DOI 10. 18307/2021. 0419

(c) 2021 by Journal of Lake Sciences

\title{
气候变化和人类活动对鄱阳湖水龄影响的定量区分”
}

\author{
范宏翔 ${ }^{1}$, 徐力刚 ${ }^{1,3 * *}$, 朱 华 ${ }^{1,2}$, 鲁 照 ${ }^{1,4}$, 曹宇贤 ${ }^{1,2}$, 吴亚坤 ${ }^{1,4}$, 姜加虎 ${ }^{1}$ \\ (1: 中国科学院南京地理与湖泊研究所,中国科学院流域地理学重点实验室,南京 210008) \\ (2: 中国科学院大学, 北京 100049) \\ (3:中国长江三峡集团有限公司长江生态环境工程研究中心, 北京 100038) \\ ( 4 : 安徽工业大学能源与环境学院,马鞍山 243000)
}

\begin{abstract}
摘 要: 在气候变化和人类活动的双重影响下,湖泊内部的水动力条件正发生重大的变化,进而影响到湖泊水环境的变 化. 水龄是颗粒物从人口传输到指定点的时间, 可以定量反映水体的运动和交换程度以及滞留情况. 如何定量区分人类 活动与气候变化对水龄的影响程度, 对湖泊水资源科学管理和水环境的治理有着重要的科学意义. 本文耦合了深度学习 网络和传统二维水动力模型, 通过引人基准期概念, 定量区分了气候变化和人类活动对鄱阳湖湖区水龄变化的贡献程度. 结果表明: (1) 本文构建的鄱阳湖流域降雨一径流和鄱阳湖湖区二维水动力耦合模型, 能够较好地反映鄱阳湖湖区水量交 换及流域产流过程, 其纳西效率系数分别达到了 0.96 和 0.90 以上; (2) 鄱阳湖湖区水龄的空间差异显著, 东部湖区和南 部湖区尾问水龄平均为 $228.01 \mathrm{~d}$, 而大部分通江水体水龄较小, 平均为 $24.21 \mathrm{~d}$; (3) 在气候变化的影响下, 鄱阳湖年均有 $2054 \mathrm{~km}^{2}$ 的水体水龄呈现减少的趋势, 减小幅度为 $30 \mathrm{~d}$ 左右, 而在人类活动的影响下, 在涨水期和丰水期有约 $58 \%$ 的湖 区水龄呈现上升趋势, 而在枯水期和退水期, 平均有 $82 \%$ 的水体水龄呈现下降趋势. 研究结果能够为鄱阳湖水资源管理 和水环境治理提供技术支撑, 同时也为定量区分人类活动和气候变化对湖泊水动力的影响机制研究提供了一种新思路 和视角.
\end{abstract}

关键词: EFDC 模型;气候变化;人类活动; 鄱阳湖; 水龄

\section{Distinguishing the relative impacts of climate change and anthropogenic activities on vari- ation of water age in the Lake Poyang*}

Fan Hongxiang $^{1}$, Xu Ligang ${ }^{1,3 * *}$, Zhu Hua ${ }^{1,2}$, Lu Zhao ${ }^{1,4}$, Cao Yuxian ${ }^{1,2}$, Wu Yakun ${ }^{1,4}$ \& Jiang Jiahu ${ }^{1}$

(1: Key Laboratory of Watershed Geographic Sciences, Nanjing Institute of Geography and Limnology, Chinese Academy of Sciences, Nanjing 210008, P.R.China)

(2: University of Chinese Academy of Sciences, Beijing 100049, P.R.China)

(3: China Three Gorges Corporation, Eco-Environmental Engineering Research Center, Beijing 100038, P.R.China)

(4: School of Energy and Environment, Anhui University of Technology, Maanshan 243000, P.R. China)

Abstract: The hydrodynamics inside a lake suffers great shifts due to the combined impacts of climate change and anthropogenic activities, which in turn influences the water environment in the lake. Distinguish the impacts of climate change and anthropogenic activities on water age, which indicates the flow patterns and water exchange rates, is of great significance to watershed management. This study performs a quantitative attribution analysis by coupling the environmental fluid dynamics code( EFDC) and the LSTM model. Results showed that the model used in this study can accurately simulate the water level and runoff dynamics, with the Nash-Sutcliffe Efficiency over 0.96 and 0.90 , respectively. (2) There exits great spatial heterogeneity of the water age, with

* 2020-10-21 收稿;2020-12-11 收修改稿.

国家重点研发计划项目 (2018YFE0206400, 2018YFC0407606)、国家自然科学基金项目 (42001109,41971137)、江 苏省自然科学基金项目 (BK20201102)、中国长江三峡集团有限公司项目 (201903145)、中国科学院南京地理与湖 泊研究所引进人才启动项目 ( NIGLAS2019QD005)、中国科学院流域地理学重点实验室开放基金项目 (WSGS2020003) 和四川省教育厅课题 (17ZB0399) 联合资助.

** 通信作者;E-mail: lgxu@ niglas.ac.cn. 
average water age of 228.01 and 24.21 days in the floodplains and main channel, respectively. (3) The water age decreased about 30 days for over $2054 \mathrm{~km}^{2}$ due to climate change, whereas anthropogenic activities increase the water age in $58 \%$ lake area in the dry period and shorten the water age in $82 \%$ lake area in the flood period. The results can provide scientific basis and theoretical guidance for water resources management in Lake Poyang Basin.

Keywords: Environmental fluid dynamics code (EFDC); climate change; human activities; Lake Poyang; water age

随着全球气候变化的加剧,世界各国均面临着不同程度的水资源危机. 全球水资源的管理是 21 世纪最 重要的挑战之一. 世界上最大的水资源问题是水资源短缺. 气候变化和人类活动正显著影响着全球和区域 水文循环过程,成为导致水文水资源时空分布不均的重要因素; 同时也是流域一湖泊水文情势变化的主要原 因 ${ }^{[1]}$. 在气候变化和人类活动的双重影响下, 湖泊内部的水动力条件也发生了重大的变化, 湖泊水动力和富 营养化往往存在胁迫一响应关系,湖泊水动力的演变势必给湖泊水环境带来影响.

数值模拟被认为是预测水体中污染物的迁移转化的重要手段之一 ${ }^{[2-3]}$. 截至目前, 已有大量研究依托数 值模型在鄱阳湖开展水环境的研究工作 (表 1 ), 如李冰 ${ }^{[4]}$ 基于 MIKE21 构建鄱阳湖二维物理过程模型, 研究 了水情条件变化对湖泊防洪、水资源、水环境, 乃至湖泊总体水安全的影响, 结果表明影响鄱阳湖水安全状 况的主要因素是流域来水变化、长江干流来水变化、流域人湖污染物通量和湖区人类活动. 最近, 国内部分 学者针对设想中的鄱阳湖水利枢纽工程对鄱阳湖湖区水环境的可能影响进行了模拟预测研究, 结果表明在 水利枢纽不同运用方案下, 枯水期主湖区的总氮和总磷浓度低于现状, 但尾问区域污染物浓度显著升高, 加 之湖流较现状条件下变缓, 鄱阳湖局部地区的富营养化风险增加 ${ }^{[5]}$. 综上, 大多数研究主要着眼于鄱阳湖水 环境本身的变化及外部其影响因素, 而对于其内在机制的研究较少. 特别是在气候变化和人类活动共同作 用下,湖泊内部的水动力条件也发生了巨大的变化,其对水环境的影响机制尚不清楚.

表 1 鄱阳湖数值模拟研究进展

Tab.1 Summary of the applications of numeric modeling in Lake Poyang

\begin{tabular}{|c|c|c|c|}
\hline 应用模型 & 模拟对象 & 主要研究内容 & 参考文献 \\
\hline EFDC & 水动力 + 水质 & 鄱阳湖水质模拟不确定性 & 张庆合 ${ }^{[6]}$ \\
\hline EFDC & 水动力 & 鄱阳湖水动力特征 & 熊雄[7] \\
\hline EFDC & 水动力 & 鄱阳湖水利枢纽工程影响评价 & Lai 等 ${ }^{[8]}$ \\
\hline EFDC & 水动力 + 水质 & 湖泊水生态系统健康评价 & 齐凌艳 ${ }^{[9]}$ \\
\hline EFDC & 水动力+水质 & 鄱阳湖富营养化响应机制 & 黄爱平 [10] \\
\hline WASP7 & 水质 & 鄱阳湖水质预测与防控措施 & 张玲霞 ${ }^{[11]}$ \\
\hline WATLAC+MIKE21 & 水文+水动力 & 鄱阳湖流域系统联合模拟 & 李云良 ${ }^{[12]}, \mathrm{Li}$ 等 ${ }^{[13]}$ \\
\hline SWAT+H3D-PYH & 水质 & 鄱阳湖水质 & 邹友琴等 ${ }^{[14]}$ \\
\hline MIKE21 & 水动力 & 湖泊水文情势变化影响因素 & 李梦凡 ${ }^{[15]}$ \\
\hline MIKE21 & 水动力+水质 & 湖泊水安全评价 & 李冰 ${ }^{[4]}$ \\
\hline 自主开发二维水质模型 & 水动力+水质 & 鄱阳湖水质变化影响因素 & Wang 等 ${ }^{[5]}$ \\
\hline MIKE21 & 水动力 & 鄱阳湖极端水情分析 & Zhang 等 ${ }^{[16]}$ \\
\hline СHAM & 水动力 & 三峡工程对鄱阳湖的影响 & Lai 等 ${ }^{[17]}$ \\
\hline
\end{tabular}

为了定量认识流场在物质输运中的作用, 有必要研究与流场密切相关又能反映其本质的辅助变量, 比 如水龄, 进而通过研究这些辅助变量, 有助于人们更好地理解湖泊水动力过程的环境效应. 水龄作为换水能 力的一种重要表达, 可以定量反映湖泊水体的交换能力和滞留状况, 对评估水质的变化以及富营养化风险 识别具有重要意义 ${ }^{[10,18]}$. 鄱阳湖的水龄研究较少, 并且也大多都集中在水龄对水环境的影响方面, 如黄爱 平 ${ }^{[10]}$ 就基于水龄提出了鄱阳湖富营养区域的识别方法; 范宏翔 ${ }^{[19]}$ 则发现水文情势对鄱阳湖水质的影响主 要通过影响湖泊的水龄来体现. 但是, 对于水龄本身变化及其影响因素的研究较少. 气候变化和人类活动影 响着全球和区域水文循环过程, 是导致水文水资源时空分布不均的重要因素, 同时也是流域一湖泊水文情势 变化的主要原因 ${ }^{[20]}$. 定量区分人类活动与气候变化对湖区水动力条件的影响分量, 对于湖区水环境污染风 
险识和预警别具有重要的科学意义. 目前, 已有大量研究区分了二者对流域水文情势的影响 ${ }^{[21-23]}$, 然而对于 气候变化和人类活动对湖区内部水动力条件的影响研究则十分缺乏, 这主要是由于其对水文数据有着较高 的要求, 需要不同情景下长时间尺度连续的水位流量数据. 近期, 深度学习网络的发展解决了时间序列的长 短期依赖问题, 大大提高了时间序列预测的精度. 其在水文领域的应用也越来越广泛 ${ }^{[24-27]}$, 利用深度学习网 络构建流域径流预测模型, 并通过与历史时期的数据进行对比, 可以得到精细的时间尺度上的径流变化数 据, 并为进一步驱动水动力模型提供数据基础.

综上所述, 本文目的旨在 (1) 构建鄱阳二维水动力模型与气象径流模型, 模拟计算鄱阳湖水龄的时空变 化过程; (2) 通过引人基准期概念, 并耦合构建的二维水动力模型与气象径流模型, 利用差异化的情景模拟, 定量区分鄱阳湖水龄改变的主要影响因素. 研究结果能够揭示鄱阳湖水环境对水文水动力变异的响应, 为 鄱阳湖水污染防治提供科学支持, 同时为流域水资源管理提供一种新的思路和方法.

\section{1 材料与方法}

\section{1 基础数据}

根据资料搜集情况,选取鄱阳湖流域时间序列最长的 14 个国家级气象站(图 1) 的日气象数据(包括降 雨、温度、气压、湿度、日照时数、风速、饱和水汽压等), 数据时段为 $1960-2015$ 年. 鄱阳湖人湖水量主要来 自 “五河”, 分别为赣江、抚河、信江、饶河和修水. 选取 5 个子流域下游出口断面流量监测站 (图 1) 日平均径 流数据来训练和验证鄱阳湖流域降雨一径流模型.
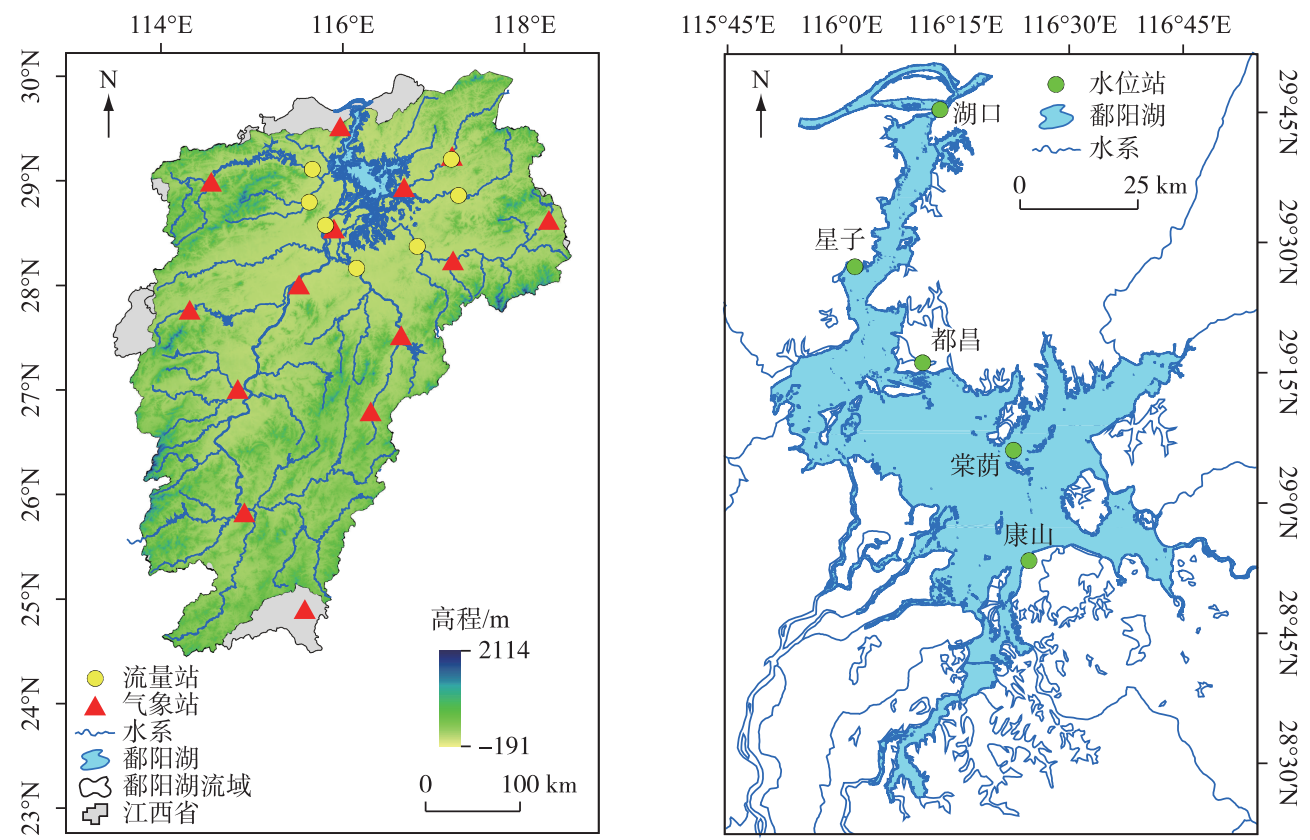

图 1 鄱阳湖流域地理位置及气象站分布

Fig. 1 Location of Lake Poyang Basin and the meteorological stations inside the basin

\section{2 研究方法}

1.2.1 鄱阳湖流域气象一径流模型构建 基于长短记忆模型框架构建神经网络模型来模拟鄱阳湖流域的径 流过程. 构建的模型有一个输人层, 共包含 128 个 LSTM 神经元, 用来输人流域的气象参数. 在此模型中, 输 人层为经过标准化后的鄱阳湖流域 14 个国家气象站点的逐日观测数据,包括日降雨、日平均气温、日最高 气温、日最低气温、日平均气压、太阳辐射、相对湿度、极大风速以及近地面风速. 另外, 为了防止模型出现过 拟合的现象, 在输人层之后设置了一个丢弃层, 并设置其丢弃率为 $0.4^{[28]}$. 模型输出层是一个包含 5 个独立 
输出端的全连接层. 输出数据为经过标准化后的赣江、抗河、信江、饶河和修水的逐日径流过程. 模型主要的 参数类型包括全局超参数、训练时超参数以及普通参数等, 其中, 全局超参数以及训练时超参数通过经验值 进行设定, 普通参数在模型训练时进行自动优化. 模型结构及主要参数设定参见范宏翔等 ${ }^{[29]}$.

1.2.2 鄱阳湖湖区水动力模型构建 本文采用 EFDC( environmental fluid dynamics code) 模型构建鄱阳湖湖区 二维水动力模型. EFDC 模型是美国环境保护局推荐使用的水环境生态模型之一 ${ }^{[30]}$, 最早由美国弗吉尼亚 海洋研究所开发 ${ }^{[31]}$, 其物理机制和很多计算方法借鉴了曾被广泛使用的 Blumberg-Mellor 模型 ${ }^{[32]}$ 和美国陆 军工程兵团开发的切萨皮克湾模型 ${ }^{[33]}$. EFDC 模型包括了水动力、风浪、泥沙、富营养化、有毒物质和沉积物 多个模块,并良好地耦合为一个整体 ${ }^{[30]}$, 可以进行水体一维、二维和三维流场、盐分、黏性和非黏性泥沙输 运、生态过程和淡水人流的模拟. 模型在垂直方向采用 $\sigma$ 坐标变换, 水平方向采用直角或者曲线正交坐标 系. 水动力模块是 EFDC 模型的核心, 其水动力方程采用有限差分法进行求解, 水平方向采用交错网格离 散, 时间采用二阶精度的有限差分法和内外模式分裂法进行积分. 其中, 外模块采用半隐式格式, 允许较大 的时间步长; 内模块使用垂直扩散的隐式格式. EFDC 在潮间带区域采用干湿网格技术, 能够很好的刻画水 面的干湿变化过程. EFDC 模型应用广泛, 能够模拟河流、河口、湖泊、水库、湿地和自近岸到陆架的海域, 并 能同时考虑风、浪、潮、径流和水工建筑物的影响.

1.2.3 模型基本设定和概化 在模型构建的基本设定和概化思想中,应充分考虑以下因素: (1) 鄱阳湖湖底 地形起伏较大, 湖中主河道形状弯曲且深度较大, 水动力条件复杂, 网格构建时应该对主河道区域有足够的 刻划; (2) 鄱阳湖属于亚热带湿润季风气候区, 有较为明显的季风特征, 春季和冬季受到西伯利亚冷气流的 影响, 盛行偏北风; 夏季和秋季受到太平洋副热带高压控制, 盛行偏南风, 多年平均风速为 $3.01 \mathrm{~m} / \mathrm{s}$. 尽管风 作为影响鄱阳湖水文情势变化的次要因素, 对整体水位模拟效果和精度影响较小, 但是风场也是湖泊水动 力过程的重要驱动力, 在某些时刻对湖泊水流结构会产生重要影响 ${ }^{[34]}$. 因此, 在构建鄱阳湖二维水动力模 型时, 必然要考虑风场条件对鄱阳湖水动力模拟过程的影响; (3) 由于鄱阳湖 “高水是湖, 低水似河” 的独特 水文特性, 在构建鄱阳湖二维水动力耦合模型时, 认为水动力指标在垂向近似均匀, 以垂向平均值表达 ${ }^{[8]}$. 鄱阳湖流域气象径流模型和湖区二维水动力模型耦合过程如图 2 所示.

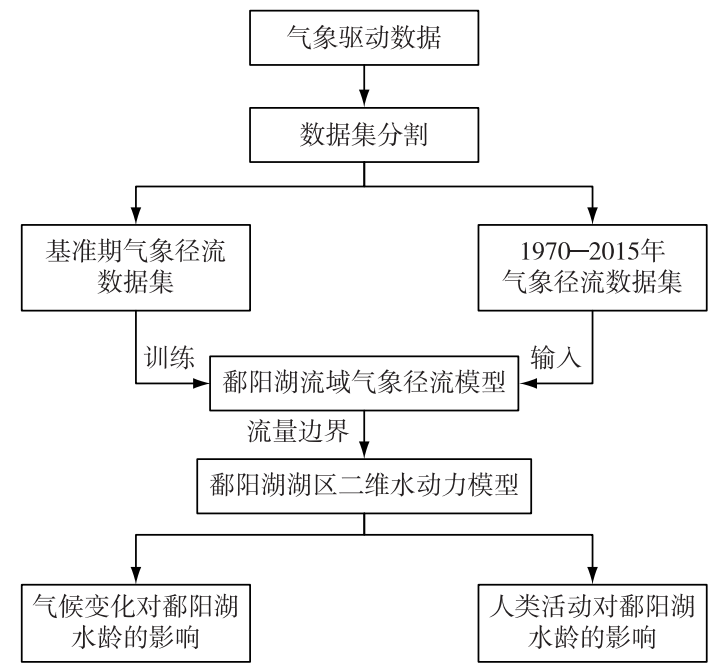

图 2 鄱阳湖气象径流模型和水动力模型耦合框架

Fig.2 Framework of coupling LSTM runoff model and EFDC hydrodynamic model

1.2.4 模型地形网格剖分 模型采用 2010 年鄱阳湖湖盆地形原始资料(基面为 85 国家高程),通过导人基于 测量和数字化的地形数据来设置湖盆地形. 模型计算域边界则根据鄱阳湖淹没范围确定, 在水平方向将计 算区域或划分为 16736 个计算单元, 采用曲线一正交网格,单元尺度为: $i$ 方向 (东西向)约 $56 \sim 1578 \mathrm{~m}, j$ 方向 (南北向) 约 30 1420 m. 由于鄱阳湖湖区地形起伏较大, 为了更准确地模拟鄱阳湖的水动力过程, 对鄱阳湖 
主河道区域的网格进行了局部加密. 主河道区域的网格分辨率大致为 $30 \mathrm{~m} \times 60 \mathrm{~m}$ (图 3).
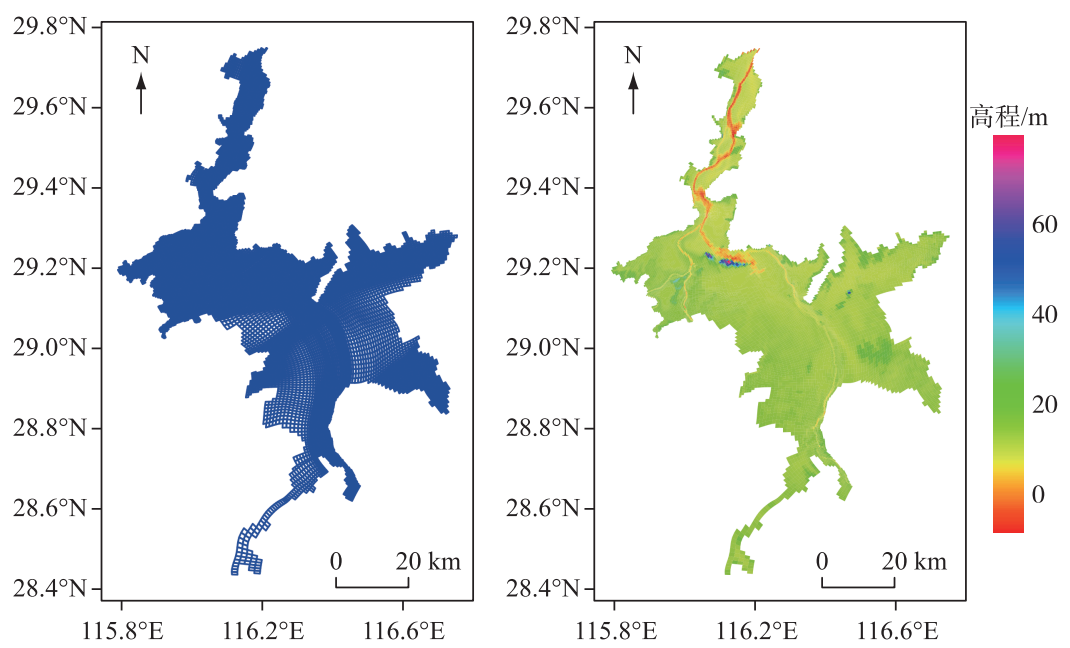

图 3 模型所用地形和计算网格

Fig.3 Bottom elevation and computational grids of the model

1.2 .5 模型初始条件与边界条件 模型选取 2012 年全年水位、水质过程进行率定验证. 为了减少初始条件对 模型的影响, 尤其是初始流速场对模拟结果的影响, 在本研究中, 模型采用热启动的方式进行启动, 即启动 前先预热 1 个月,水动力和水质模块的预热期为 2011 年 12 月 1 日至 12 月 31 日. 正式模拟时, 水动力模型 的初始水位场和流速场采用预热后的水位场及流速场作为输人. 鄱阳湖的主要人湖河流共有 5 条(简称“五 河”). 本模型共定义了 8 个开边界, 其中包括 7 个流量边界和 1 个水位边界. 7 个流量边界作为水动力模型 的上游开边界, 分别为赣江主支、赣江中支、赣江南支、抚河、信江、皆河和修水,其表征了流域来水对湖泊水 文水动力水环境的影响. 将北部湖泊出口一湖口的水位过程线作为模型的下游开边界, 用来表征鄱阳湖与长 江之间的出流、顶托和倒灌效应. 上游各个开边界的流量过程根据五河尾问的主要控制站 (外洲、李家渡、梅 港、渡峰坑、虎山、虬津和万家埠) 和各河道从控制站到入湖的传播时间给定 ${ }^{[4]}$. 其中, 赣江各条支流流量根 据其占外洲站流量的份额按经验分配. 此外, 由于鄱阳湖有 $25000 \mathrm{~km}^{2}$ 的区域属于无站点控制的集水区,为 保证湖泊水量平衡, 提高水动力模块的准确性和合理性,需要合理估算未控区间的产流过程, 并与流域 “五 河” 控制站的流量过程共同作为模型的上游边界条件 ${ }^{[4,15]}$. 本文采用降雨一径流系数法对其进行估算:

$$
W=P \cdot \Delta A \cdot a
$$

式中, $W$ 表示某年未控区间的产流总量 $\left(\mathrm{m}^{3}\right) ; P$ 表示该年降雨总量 $\left(\mathrm{m}^{3}\right) ; \Delta A$ 表示鄱阳湖流域未控区间的集 水区面积 $\left(\mathrm{km}^{2}\right) ; a$ 表示鄱阳湖流域的评价径流系数, 在本文中取经验值 $0.6^{[35]}$.

1.2.6 模型评价指标 模型的模拟效果采用常规的统计指标来量化评价, 在本研究中主要采用的指标为纳什 效率系数 $(N S E)$ 、均方根误差 $(R M S E)$ 以及平均绝对误差 $(M A E)$, 其计算公式分别为:

$$
\begin{aligned}
& N S E=1-\frac{\sum_{i=1}^{n}\left(y_{i}-y_{i}^{\prime}\right)^{2}}{\sum_{i=1}^{n}\left(y_{i}-\bar{y}\right)^{2}} \\
& R M S E=\sqrt{\frac{\sum_{i=1}^{n}\left(y_{i}-y_{i}^{\prime}\right)^{2}}{n}} \\
& M A E=\frac{1}{n} \sum_{i=1}^{n}\left|y_{i}-y_{i}^{\prime}\right|
\end{aligned}
$$

式中, $y^{\prime}$ 为模拟序列, $y$ 为观测序列, $y$ 为观测序列均值, $n$ 为序列长度. 
1.2.7 模型数据可视化 基于 $\mathrm{R}$ 语言中的 ncdf4 、 data.table 以及 tidyverse 软件包, 独立开发了 EFDC 模型前后 处理软件包 efder(https://hxfan1227.github.io/efdcr/). efdcr 在极大程度解决了 EFDC 模型结果的可视化问 题, 具有极强的灵活性. 同时, efder 还提供了一系列工具函数来辅助进行 EFDC 的模型构建过程. efder 的主 要功能和工作流程如图 4 所示.

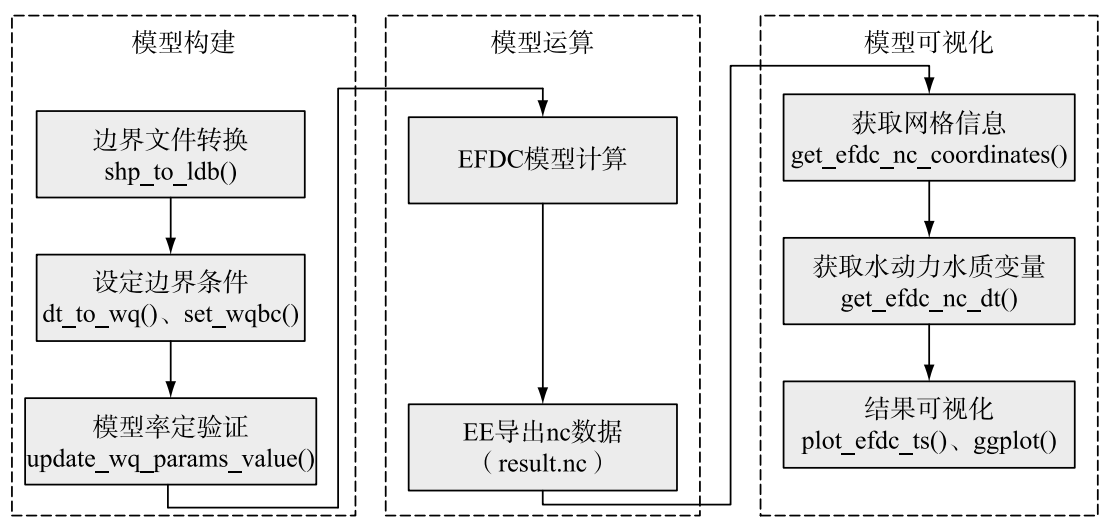

图 4 efder 工作流程

Fig.4 The workflow of efdcr

1.2.8 情景设置方案 为了定量区分气候变化和人类活动对鄱阳湖水龄变化的贡献率, 本文设置了 3 个情 景. S0 作为基准情景, 即自然状况下鄱阳湖流域的径流过程, 在设置时参考前人的研究, 将 1960-1969 年作 为基准期 ${ }^{[23,36-37]}$. 同时,考虑到 LSTM 模型的特殊性,基准期选取时也考虑了不同的流域来水情景. 根据水文 情报预报规范 (GB/T 22482-2008), 按照鄱阳湖流域年最大流量进行水文年的划分. 其中, 1962 年为典型 丰水年, 1963 年为典型枯水年, 1966 年和 1968 年为典型平水年. 因此, 我们认为选用 1960-1969 作为基准 期, 能够满足情景模拟的需要. 为了进一步区分人类活动与气候变化对鄱阳湖径流的影响, 在模型训练和验 证时仅使用基准期的气象水文数据, 为了防止数据泄露问题, 将基准期划分为训练期和验证期两个阶段: 模 型的训练周期从 1960 年 1 月 1 日至 1968 年 12 月 31 日,共计 3287 d. 模型的验证周期从 1969 年 1 月 1 日至 1969 年 12 月 31 日,共计 365 d. S 1 鄱阳湖气象一径流模型模拟的 1970-2015 年鄱阳湖流域 “五河” 多年平 均日流量过程,S2 为实测的 1970-2015 年鄱阳湖流域“五河” 多年平均日流量过程. 通过对比 S1 和 S0, 可 以定量区分气候变化对鄱阳湖水龄变化的影响分量; 而通过对比 S2 和 $\mathrm{S} 1$, 则可以定量区分人类活动对鄱阳 湖水龄变化的影响贡献.

1.2.9 影响程度计算 参照 $\mathrm{Ye}$ 等 ${ }^{[23]}$ 关于定量区分气候变化和人类活动对鄱阳湖流域径流量影响程度的分 析方法,由气候变化和人类活动引起的鄱阳湖水龄变化可以用下式来解释:

$$
\left\{\begin{array}{l}
\Delta W A_{\mathrm{C}}=W A_{1}-W A_{0} \\
\Delta W A_{\mathrm{H}}=W A_{2}-W A_{1}
\end{array}\right.
$$

式中, $W A_{0} 、 W A_{1}$ 和 $W A_{2}$ 分别代表 S0、S1 以及 $\mathrm{S} 2$ 情景下鄱阳湖水龄的变化过程, $\Delta W A_{\mathrm{C}}$ 和 $\Delta W A_{\mathrm{H}}$ 分别代表气 候变化和人类活动引起的鄱阳湖水龄变化的绝对分量.

\section{2 研究结果}

\section{1 鄱阳湖气象径流模型验证}

训练期模型在各个子流域的 NSE 分别为赣江 0.94 、抚河 0.95 、信江 0.95 、饶河 0.95 和修水 $0.94 ; R M S E$ 分别为贑江 $630.80 \mathrm{~cm}^{3} / \mathrm{s}$ 、抚河 $143.64 \mathrm{~cm}^{3} / \mathrm{s}$ 、信江 $194.74 \mathrm{~cm}^{3} / \mathrm{s}$ 、饶河 $129.46 \mathrm{~cm}^{3} / \mathrm{s}$ 和修水 $144.01 \mathrm{~cm}^{3} / \mathrm{s}$. 而 在验证期, 模型在各个子流域的 NSE 分别为贑江 0.90 、抚河 0.95 、信江 0.95 、饶河 0.98 和修水 $0.96 ; R M S E$ 分 别为贑江 $671.03 \mathrm{~cm}^{3} / \mathrm{s}$ 、抚河 $158.90 \mathrm{~cm}^{3} / \mathrm{s}$ 、信江 $212.04 \mathrm{~cm}^{3} / \mathrm{s}$ 、饶河 $139.52 \mathrm{~cm}^{3} / \mathrm{s}$ 和修水 $162.79 \mathrm{~cm}^{3} / \mathrm{s}$ (表 2 ). 因此可以认为构建的气象一径流模型能够很好地模拟鄱阳湖流域的径流过程. 
表 2 鄱阳湖流域气象一径流模型模拟表现

Tab.2 Simulation performance of LSTM runoff model in Lake Poyang Basin

\begin{tabular}{|c|c|c|c|c|c|c|}
\hline \multirow{2}{*}{ 流域 } & \multicolumn{3}{|c|}{ 训练期 } & \multicolumn{3}{|c|}{ 验证期 } \\
\hline & $R M S E /\left(\mathrm{cm}^{3} / \mathrm{s}\right)$ & NSE & $R^{2}$ & $R M S E /\left(\mathrm{cm}^{3} / \mathrm{s}\right)$ & NSE & $R^{2}$ \\
\hline 赣江 & 630.80 & 0.94 & 0.94 & 671.03 & 0.90 & 0.89 \\
\hline 抚河 & 143.64 & 0.95 & 0.95 & 158.90 & 0.95 & 0.95 \\
\hline 信江 & 194.74 & 0.95 & 0.95 & 212.04 & 0.95 & 0.95 \\
\hline 饶河 & 129.46 & 0.95 & 0.95 & 139.52 & 0.98 & 0.98 \\
\hline 修水 & 144.01 & 0.94 & 0.94 & 162.79 & 0.96 & 0.96 \\
\hline
\end{tabular}

\section{2 鄱阳湖二维水动力模型验证}

验证选取了鄱阳湖湖区 4 个水文站点 (自北向南依次为星子、都昌、棠荫和康山). 验证时段选择平水年 2012 年 1 月 1 日至 12 月 31 日. 其中, 水位验证的结果如图 5 所示. 如图所示, 本文构建的鄱阳湖水环境模 型的水动力模块模拟效果良好,其模拟的水位序列与实测序列吻合度较高.
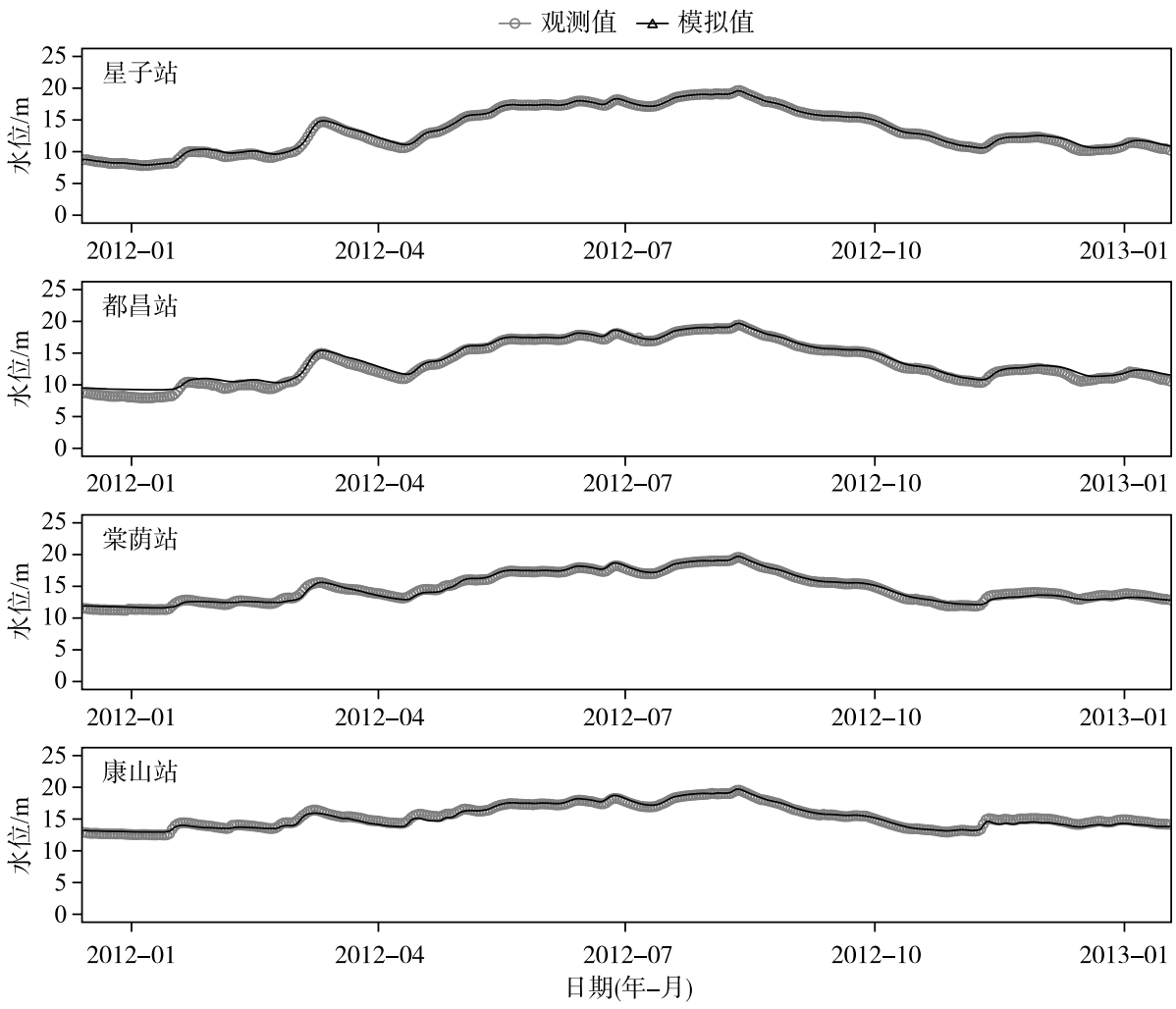

图 5 鄱阳湖湖区各水位站水位验证结果

Fig.5 Comparison of simulated and measured water level in Xingzi, Duchang, Tangying and

Kangshan Staions in Lake Poyang

由鄱阳湖 2012 年水位模拟精度可以发现, 4 个水文站点水位模拟的决定系数 $\left(R^{2}\right)$ 均在 0.97 以上, 均方 根误差 $(R M S E)$ 在 $0.30 \sim 0.58 \mathrm{~m}$ 之间, 纳什效率系数 $(N S E)$ 均在 0.96 以上. 平均绝对误差 $(M A E)$ 在 $0.20 \sim$ $0.50 \mathrm{~m}$ 之间 (表 3 ). 综上所述, 本文构建的鄱阳湖水动力模块能够较好地反映鄱阳湖湖区水量交换及平衡 的季节性变化特征, 模拟结果真实可靠. 
表 32012 年鄱阳湖水位模拟精度

Tab.3 Simulation accuracy of water level in Lake Poyang in 2012

\begin{tabular}{ccccc}
\hline 水位站 & $M A E / \mathrm{m}$ & $N S E$ & $R^{2}$ & $R M S E / \mathrm{m}$ \\
\hline 星子 & 0.20 & 0.99 & 1.00 & 0.30 \\
都昌 & 0.50 & 0.97 & 0.99 & 0.58 \\
棠荫 & 0.28 & 0.98 & 0.98 & 0.32 \\
康山 & 0.31 & 0.96 & 0.97 & 0.36 \\
\hline
\end{tabular}

\section{3 基准情景下鄱阳湖水龄的时空分布特征}

自然条件下 $(\mathrm{S} 0)$ 鄱阳湖水龄的时空分布特征如图 6 所示. 可以发现, 鄱阳湖湖区的水龄存在明显的空 间分异特征,其最主要特征为东部湖区和南部湖区尾问水龄较大,平均为 $228.01 \mathrm{~d}$,而大部分通江水体水龄 较小, 平均为 $24.21 \mathrm{~d}$. 在洪泛湿地区域, 水龄有比较明显的时间差异. 枯水期洪泛湿地与主湖断开连接, 形 成独立的水体, 导致其水龄增大, 年平均水龄约为 $90 \mathrm{~d}$ 左右. 而在丰水期, 随着水位上涨, 洪泛湿地逐渐与主 湖连接,形成一个整体,其水龄逐渐减小,平均为 $30 \mathrm{~d}$ 左右.

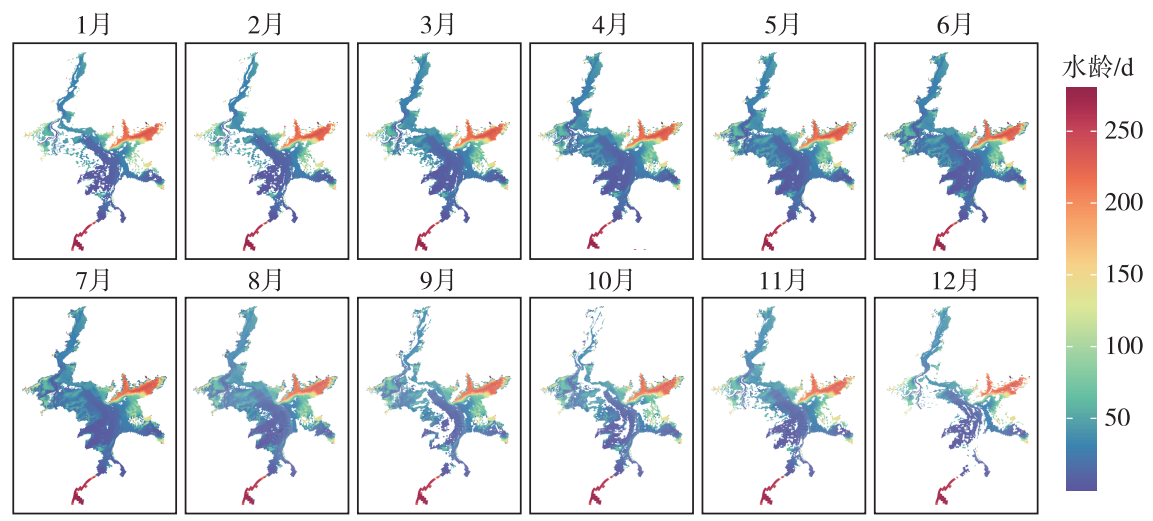

图 6 自然条件下 $(\mathrm{S} 0)$ 鄱阳湖水龄时空分布特征

Fig. 6 The spatial-temporal distribution pattern of water age in Lake Poyang under S0 scenario

\section{4 气候变化与人类活动对鄱阳湖水龄的影响分析}

图 7 和表 4 为气候变化对鄱阳湖水龄影响程度的时空差异 $(\mathrm{S} 1 \sim \mathrm{S} 0)$ 和影响范围统计, 由图 7 可以发现, 在气候变化的影响下, 鄱阳湖全湖大部分湖区的水龄都呈现下降的趋势, 下降幅度较小, 约为 $20 \%$ 左右; 而 在湖区东部,有部分区域水龄呈现上升的趋势,最大上升幅度超过 $100 \%$.

从影响范围来看 (表 4), 在气候变化影响下, 年均有 $2054 \mathrm{~km}^{2}$ 的水体水龄呈现减少的趋势, 但是减少幅 度较小; 相反,年均有 $736 \mathrm{~km}^{2}$ 的水体水龄呈现上升的趋势, 上升幅度约在 $30 \mathrm{~d}$ 左右.

表 4 气候变化对鄱阳湖水龄影响范围统计 $\left(\mathrm{km}^{2}\right)$

Tab.4 The influenced area related to several water age changes of Lake Poyang caused by climate change $\left(\mathrm{km}^{2}\right)$

\begin{tabular}{ccccccccccccc}
\hline 水龄变化 $\mathrm{d}$ & 1 月 & 2 月 & 3 月 & 4 月 & 5 月 & 6 月 & 7 月 & 8 月 & 9 月 & 10 月 & 11 月 & 12 月 \\
\hline$(-128,-96]$ & 0 & 0 & 0 & 0 & 0 & 0 & 0 & 0 & 0 & 0 & 0 & 4 \\
$(-96,-64]$ & 0 & 0 & 0 & 0 & 0 & 0 & 0 & 0 & 0 & 0 & 0 & 10 \\
$(-64,-32]$ & 0 & 0 & 3 & 0 & 0 & 1 & 0 & 0 & 0 & 4 & 101 & 129 \\
$(-32,0]$ & 1797 & 1910 & 2140 & 2158 & 1046 & 1936 & 1292 & 2839 & 2844 & 2758 & 2203 & 1725 \\
$(0,32]$ & 1219 & 471 & 455 & 690 & 1923 & 1141 & 1802 & 228 & 187 & 222 & 255 & 235 \\
$(32,64]$ & 0 & 0 & 0 & 0 & 0 & 0 & 0 & 0 & 0 & 1 & 0 & 1 \\
\hline
\end{tabular}




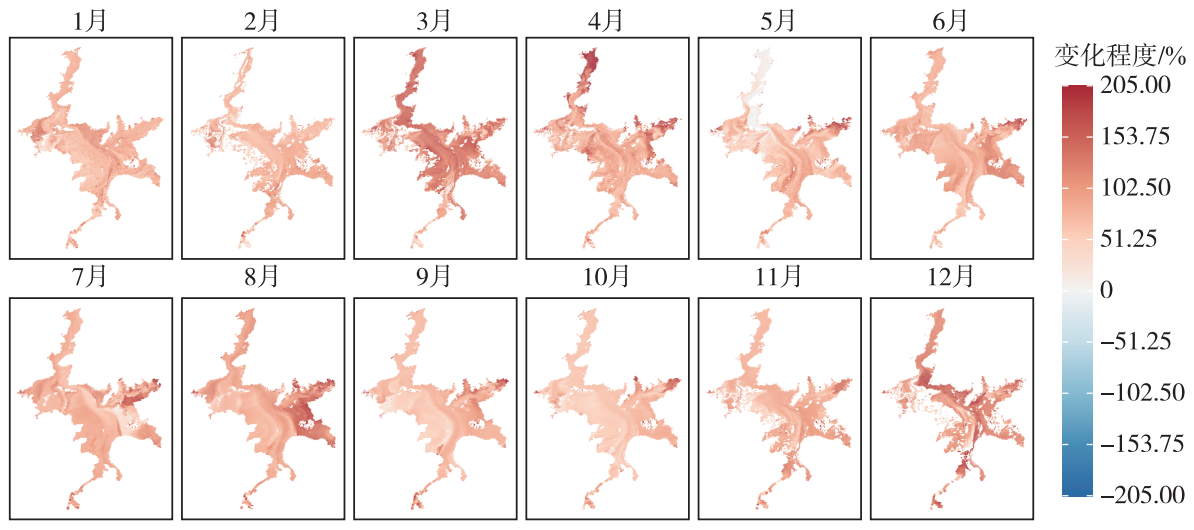

图 7 气候变化对鄱阳湖水龄影响程度的时空差异

Fig.7 The spatial-temporal distribution pattern of impacts of climate change on water age in Lake Poyang

进一步区分了人类活动对鄱阳湖水环境的影响程度. 人类活动的影响计算方式为 $(\mathrm{S} 2-\mathrm{S} 1) /(\mathrm{S} 2-\mathrm{S} 0)$. 图 8 和表 5 为人类活动对鄱阳湖水龄影响的时空差异和影响范围统计. 由图 8 可以发现, 人类活动对鄱阳湖 水龄的影响存在明显的时空差异. 从空间上来说,在人类活动影响下,东部湖区的水龄呈现显著的下降趋 势, 最大下降幅度超过 30\%, 而中、北部湖区水体水龄则总体呈现上升的趋势, 最大上升幅度超过 $100 \%$. 其 余部分的水体水龄变化并没有呈现明显的时空特征,变化并无规律.
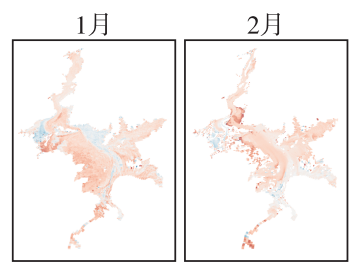

8月
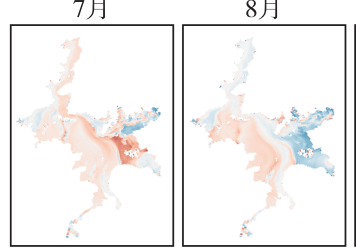

图 8 人类活动对鄱阳湖水龄影响程度的时空差异

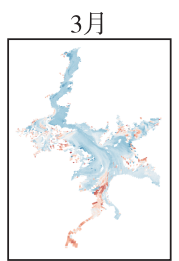

9月

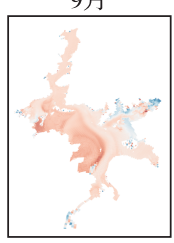

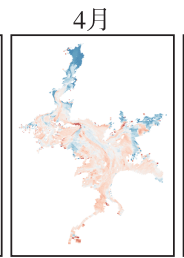

10月

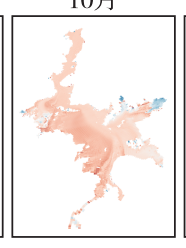

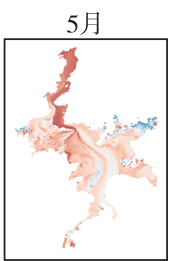

11月

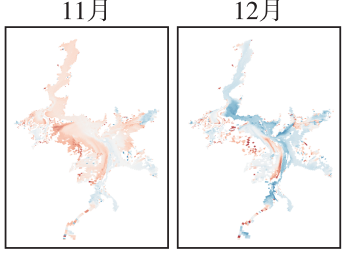

6月
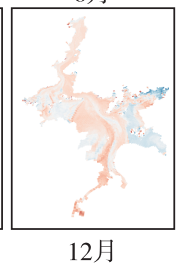

变化程度 $/ \%$

123.00

92.25

61.50

30.75

0

$-30.75$

$-61.50$

$-92.25$

$-123.00$

Fig. 8 The spatial-temporal distribution pattern of impacts of human activities on water age in Lake Poyang

表 5 人类活动对鄱阳湖水龄影响范围统计 $\left(\mathrm{km}^{2}\right)$

Tab.5 The influenced area related to several water age changes of Lake Poyang caused by human activities $\left(\mathrm{km}^{2}\right)$

\begin{tabular}{ccccccccccccc}
\hline 水龄变化 $/ \mathrm{d}$ & 1 月 & 2 月 & 3 月 & 4 月 & 5 月 & 6 月 & 7 月 & 8 月 & 9 月 & 10 月 & 11 月 & 12 月 \\
\hline$(-128,-96]$ & 0 & 0 & 0 & 0 & 0 & 0 & 0 & 0 & 0 & 0 & 0 & 0 \\
$(-96,-64]$ & 0 & 0 & 0 & 0 & 0 & 0 & 0 & 0 & 0 & 0 & 0 & 0 \\
$(-64,-32]$ & 0 & 0 & 0 & 0 & 0 & 0 & 0 & 0 & 0 & 0 & 0 & 0 \\
$(-32,0]$ & 2135 & 1801 & 337 & 424 & 308 & 813 & 845 & 2037 & 2775 & 2789 & 2011 & 566 \\
$(0,32]$ & 880 & 580 & 2262 & 2424 & 2662 & 2265 & 2248 & 1031 & 256 & 196 & 548 & 1535 \\
$(32,64]$ & 0 & 0 & 0 & 0 & 0 & 0 & 0 & 0 & 0 & 1 & 0 & 3 \\
$(64,96]$ & 0 & 0 & 0 & 0 & 0 & 0 & 0 & 0 & 0 & 0 & 0 & 0 \\
$(96,128]$ & 0 & 0 & 0 & 0 & 0 & 0 & 0 & 0 & 0 & 0 & 0 & 0 \\
\hline
\end{tabular}


从影响范围来看 (表 5), 在涨水期和丰水期 (3-7 月) 仅有 $545 \mathrm{~km}^{2}$ 的水体水龄呈现下降趋势, 占全湖水 面积的 $13 \%$,下降程度在 $0 \sim 32 \mathrm{~d}$ 之间, 同时, 有超过 $2372 \mathrm{~km}^{2}$ 的水体水龄呈现上升趋势, 约占全湖面积的 $58 \%$. 而在枯水期和退水期, 平均有 $718 \mathrm{~km}^{2}$ 的水体水龄呈现上升趋势, 占全湖面积的 $18 \%$.

\section{3 讨论}

本文耦合了深度学习网络和传统二维水动力模型, 通过引人基准期概念, 定量区分了气候变化和人类 活动对鄱阳湖湖区水龄变化的贡献程度. 在以往的研究中, 流域径流的模拟往往依托传统的水文模型, 如 $\mathrm{SWAT}^{[38]}$ 和 $\mathrm{WATLAC}^{[13]}$ 等. 但是, 流域模型构建的过程中, 所需要的数据资料庞杂, 且往往涉及环保、水文、 气象、农林各个部门 ${ }^{[38]}$, 难以全部获取, 限制了物理模型在数据缺乏地区的使用 ${ }^{[28,40]}$. 而本文提出利用流域 气象数据结合深度学习网络模拟流域径流过程, 同时与湖泊二维水动力模型进行耦合, 也能很好地反映流 域水文状况对湖泊的影响, 同时, 其大大降低了模型对流域资料的依赖性. 近年来, 随着很多高级的神经网 络框架如 Keras 和 Pytorch 被开发出来, 构建一个复杂的神经网络正在变得越来越方便, 为在更加精细的尺 度上区分气候变化和人类活动对水文变量影响提供了数据支持和方法借鉴.

尽管深度学习网络在模拟时间序列方面具有很大的优势, 模拟精度也相对较高, 但是仍有大量学者认 为此类黑箱模型因为不具备物理机制, 无法对结果进行解释 ${ }^{[41-43]}$. 最近, Kratzert 等 ${ }^{[44]}$ 通过对比 LSTM 中神 经元的状态, 可以揭示某些气象变量的控制机制, 但是合理性还有待于验证. 如何科学解释神经网络的结果 也成为了学界研究的重点. 在本研究中, 我们发现鄱阳湖水龄主要受气候变化的影响, 其相对贡献率超过了 $80 \%$.气候变化通过改变流域产流过程,进而影响湖泊水龄变化. 有研究指出流域人流是影响鄱阳湖水龄的 主要因素, 流域人流量越大则鄱阳湖水龄越小. 当流域人流增加 $20 \%$ 时, 湖口水龄均值减少 $1.15 \mathrm{~d}^{[10]}$. 在本 研究中,气候变化使得鄱阳湖水龄均呈现下降的趋势, 其根本原因可能是气候变化使得鄱阳湖流域的降雨 增加, 从而导致流域人湖径流量提高. 同时, 蒸发作为影响流域水量平衡的另一重要因素, 其变化对流域径 流的改变也有着决定性作用. 有研究显示, 近年来鄱阳湖流域的潜在蒸发呈现长期下降趋势 ${ }^{[23]}$, 而实际蒸 发也呈现出波动下降的趋势 ${ }^{[45]}$. 蒸发的下降也会在一定程度上导致鄱阳湖流域径流的增加. 综上, 在气候 变化作用下,鄱阳湖流域人湖径流量大量增加, 导致其水龄呈现下降的趋势.

本研究同时也区分了人类活动对鄱阳湖水龄变化的影响分量, 可以发现, 人类活动对鄱阳湖水龄变化 也有显著的影响. 人类活动通过影响流域来水, 从而间接影响了鄱阳湖湖区的水龄变化. 一般来说, 人类活 动对流域水文过程的影响主要表现在两个方面, 即改变流域下垫面特征以及兴建大型水利工程 ${ }^{[46]}$. 以江西 省为例, 有研究表明随着江西山江湖开发治理工程的实施 ${ }^{[47]}$, 从 $1985-2001$ 年, 全省水土流失面积从 330 万 $\mathrm{hm}^{2}$ 下降到 130 万 $\mathrm{hm}^{2}$; 全省森林覆盖率由 $31.5 \%$ 上升到 $59.7 \%$. 植被覆盖的大量增加, 使得流域径流迅速 减少, 径流的减少会导致鄱阳湖湖区水龄呈现一定的上升趋势. 同时, 我们发现人类活动对水龄变化的影响 存在一定的年内时空差异, 主要体现为枯水期水龄增大, 丰水期水龄减小. 这可能是由于水利工程的修建导 致流域径流变化呈现年内分布不均的趋势. 有研究指出 ${ }^{[48]}$, 三峡水库调度对中下游水情影响主要表现为 11 月至次年 4 月径流量加大,5-10 月径流量减小. 3 月径流量增加最大, 10 月减少最多, 9 月也减少较为明 显, 这很好地揭示了人类活动对水龄影响的年内差异. 但是不可否认的是, 本文没有考虑流域下垫面因素对 径流的影响. 尽管本文参考前人研究选取 1960-1969 年作为基准期, 来尽可能减少人类活动的影响, 但是 有研究显示, 鄱阳湖流域自 $1950 \mathrm{~s}$ 开始就经受了剧烈人类活动, 从而也对流域水循环造成了一定的影响 ${ }^{[23]}$. 同时, 鄱阳湖流域修筑有大量的水库, 这些水库的蓄水也会对流域的产流过程造成一定的影响 ${ }^{[49]}$. 在未来 的研究中, 应该综合考虑这些要素, 将流域下垫面情况进行参数化建模, 使得模型能够更加准确地反映气候 变化和人类活动对流域水文过程的影响.

\section{4 结论}

1) 本文基于 EFDC 构建的鄱阳湖二维水动力模型, 能够较好地反映鄱阳湖湖区水量交换及平衡的季节 性变化特征, 模拟结果真实可靠. 4 个水文站点水位模拟的 $R^{2}$ 均在 0.97 以上, NSE 均在 0.96 以上.

2) 鄱阳湖湖区的水龄存在明显的空间分异特征, 其最主要特征为东部湖区和南部湖区尾问水龄较大, 
平均为 $228.01 \mathrm{~d}$,而大部分通江水体水龄较小,平均为 $24.21 \mathrm{~d}$. 在时间尺度上,鄱阳湖水龄的差异主要体现 在洪泛湿地区域. 枯水期洪泛湿地年平均水龄约为 $90 \mathrm{~d}$ 左右. 而在丰水期, 洪泛湿地年平均水龄为 $30 \mathrm{~d}$ 左右.

3 ) 通过耦合鄱阳湖流域气象一径流模型与湖区水动力模型, 定量区分了人类活动与气候变化对鄱阳湖 水龄变化的贡献率. 在气候变化的影响下, 鄱阳湖年均有 $2054 \mathrm{~km}^{2}$ 的水体水龄呈现减少的趋势, 减小幅度为 $30 \mathrm{~d}$ 左右; 人类活动对水龄变化的影响存在一定的年内时空差异,在涨水期和丰水期有约 $58 \%$ 的湖区水龄 呈现上升趋势, 而在枯水期和退水期, 平均有 $718 \mathrm{~km}^{2}$ 的水体水龄呈现上升趋势, 占全湖面积的 $18 \%$. 从影响 权重来看,气候变化是造成鄱阳湖水龄变化的主要因素,其影响权重为 $84 \%$,而人类活动的影响仅占 $16 \%$.

\section{5 参考文献}

[ 1 ] Wan RR, Yang GS. Progress in the hydrological impact and flood response of watershed land use and land cover change. J Lake Sci, 2004, 16(3) : 258-264. DOI: 10.18307/2004.0311. [万荣荣, 杨桂山. 流域土地利用/覆被变化的水文效 应及洪水响应. 湖泊科学, $2004, \mathbf{1 6}(3): 258-264$.

[ 2 ] Imboden DM. Phosphorus model of lake eutrophication. Limnology and Oceanography, 1974, 19(2) : 297-304. DOI: 10. 4319/lo.1974.19.2.0297.

[ 3 ] Vollenweider RA. Scientific fundamentals of the eutrophication of lakes and flowing water, with particular reference to nitrogen and phosphorus as factors in eutrophication. OECD Paris, 1968.

[ 4 ] Li B. Water security evaluation and its associated responses with hydrological conditions in Yangtze-connected lakes: A case study of the Poyang Lake in China[Dissertation]. Beijing: University of Chinese Academy of Sciences, 2017. [李冰. 通江湖泊水安全评价及其对水情变化的响应研究——以鄱阳湖为例 [学位论文]. 北京: 中国科学院大学, 2017.]

[ 5 ] Wang P, Lai GY, Li L. Predicting the hydrological impacts of the Poyang lake project using an EFDC model. Journal of Hydrologic Engineering, 2015, 20(12) : 05015009. DOI: 10.1061/( asce) he.1943-5584.0001240.

[ 6 ] Zhang QH. Poyang lake water environment model and its uncertainty research based on EFDC[Dissertation]. Guangzhou : Guangzhou University, 2011. [张庆合. 基于 EFDC 鄱阳湖水环境模型与不确定性研究 [学位论文]. 广州: 广州大 学, 2011.]

[ 7 ] Xiong X. Hydrodynamic research of Poyang lake based on the EFDC [ Dissertation]. Nanchang: Nanchang University, 2014. [ 熊雄. 基于 EFDC 的鄱阳湖水动力研究 [学位论文]. 南昌: 南昌大学, 2014.]

[ 8 ] Lai GY, Wang P, Li L. Possible impacts of the Poyang Lake (China) hydraulic project on lake hydrology and hydrodynamics.Hydrology Research, 2016, 47 (S1) : 187-205. DOI: 10.2166/nh.2016.174.

[ 9 ] Qi LY. Assessment and simualtion of aquatic ecosystem health in Lake Poyang [ Dissertation]. Beijing: University of Chinese Academy of Sciences, 2017. [齐凌艳. 鄱阳湖水生态系统健康评价与模拟研究 [学位论文]. 北京: 中国科 学院大学, 2017.]

[10] Huang AP. The characteristics and response mechanism of hydrology and hydrodynamics and eutrophication in Poyang Lake [Dissertation]. Beijing: China Institute of Water Resources and Hydropower Research, 2018. [黄爱平. 鄱阳湖水文水动 力特征及富营养化响应机制研究 [学位论文]. 北京: 中国水利水电科学研究院, 2018.]

[11] Zhang LX. Time and space distribution characteristics of eutrophication and water quality prediction based on WASP model in Poyang lake[Dissertation]. Nanchang: Nanchang University, 2015. [张玲霞. 鄱阳湖区富营养化时空分布特征分析 及 WASP 模型水质预测 [学位论文]. 南昌: 南昌大学, 2015.]

[12] Li YL. Integrated hydrological and hydrodynamic simulation study for a complex Poyang Lake-Catchment System[Dissertation]. Beijing: The University of Chinese Academy of Sciences, 2013. [李云良. 鄱阳湖湖泊流域系统水文水动力联合 模拟研究 [学位论文]. 北京: 中国科学院大学, 2013.]

[13] Li YL, Zhang Q, Yao J et al. Hydrodynamic and hydrological modeling of the Poyang Lake catchment system in China. Journal of Hydrologic Engineering, 2014, 19(3) : 607-616. DOI: 10.1061/( asce) he.1943-5584.0000835.

[14] Zou YQ, Zhong MS, Zhou WB. Design and simulation of three-dimensional water quality simulation software for Poyang Lake. Journal of Nanchang University: Natural Science, 2012, 36(1) : 91-96. [邹友琴, 钟茂生, 周文斌. 鄱阳湖三维 水质模拟软件设计与仿真. 南昌大学学报: 理科版, 2012, 36(1) : 91-96.]

[15] Li MF. Hydrological changes in Poyang Lake since 2000 and the influencing factors[Dissertation]. Beijing: University of 
Chinese Academy of Sciences, 2017. [李梦凡. 2000 年以来鄱阳湖水文情势变化特征及影响因素研究 [学位论文]. 北京: 中国科学院大学, 2017.]

[16] Zhang Q, Ye XC, Werner AD et al. An investigation of enhanced recessions in Poyang Lake: Comparison of Yangtze River and local catchment impacts. Journal of Hydrology, 2014, 517: 425-434. DOI: 10.1016/j.jhydrol.2014.05.051.

[17] Lai XJ, Liang QH, Jiang JH et al. Impoundment effects of the three-gorges-dam on flow regimes in two China's largest freshwater lakes. Water Resources Management, 2014, 28(14) : 5111-5124. DOI: 10.1007/s11269-014-0797-6.

[18] Huang CL, Li X, Sun YY. Water age distribution of the Lake Taihu and impact of the Yangtze River to Lake Taihu Water Transfer Project on the water age. J Lake Sci, 2017, 29(1) : 22-31. DOI: 10.18307/2017.0103. [黄春琳, 李熙, 孙永 远. 太湖水龄分布特征及“引江济太”工程对其的影响. 湖泊科学, 2017, 29(1) : 22-31.]

[19] Fan HX. Attribution of the changes in water regime and water quality in Poyang Lake Basin[Dissertation]. Beijing: University of Chinese Academy of Sciences, 2019. [范宏翔. 气候变化和人类活动对鄱阳湖水文情势和水环境的影响机 制研究 [学位论文]. 北京: 中国科学院大学, 2019.]

[20] Cheng JX, Xu LG, Wang Q et al. Temporal and spatial variations of water level and its driving forces in Lake Dongting over the last three decades. J Lake Sci, 2017, 29(4) : 974-983. DOI: 10.18307/2017.0421. [程俊翔, 徐力刚, 王青等. 洞庭湖近 30a 水位时空演变特征及驱动因素分析. 湖泊科学, 2017, 29(4): 974-983.]

[21] Githui F, Gitau W, Mutua F et al. Climate change impact on SWAT simulated streamflow in western Kenya. International Journal of Climatology, 2009, 29(12) : 1823-1834. DOI: 10.1002/joc.1828.

[22] Narsimlu B, Gosain AK, Chahar BR. Assessment of future climate change impacts on water resources of upper sind river basin, India using SWAT model. Water Resources Management, 2013, 27(10) : 3647-3662. DOI: 10.1007/s11269-0130371-7.

[23] Ye XC, Zhang Q, Liu J et al. Distinguishing the relative impacts of climate change and human activities on variation of streamflow in the Poyang Lake catchment, China. Journal of Hydrology, 2013, 494: 83-95. DOI: 10. 1016/j.jhydrol. 2013.04.036.

[24] Guo J, Zhang YJ, Wang JB et al. Application of the long short-term memory networks for flood forecast. Journal of Water Resources Research, 2019, 8(1) : 24-32. DOI: 10.12677/JWRR.2019.81003. [ 郭炅, 张艳军, 王俊勃等. 长短期记忆 模型在小流域洪水预报上的应用研究. 水资源研究, 2019, 8(1): 24-32.]

[25] Guo Y, Lai XJ. Water level prediction of Lake Poyang based on long short? term memory neural network. J Lake Sci, 2020, 32(3) : 865-876. DOI: 10.18307/2020.0325. [郭燕, 赖锡军. 基于长短时记忆神经网络的鄱阳湖水位预测. 湖泊科学, $2020,32(3): 865-876$.

[26] Hu QF, Cao SY, Yang HB et al. Daily runoff predication using LSTM at the Ankang Station, Hanjing River. Progress in Geography, 2020, 39(4) : 636-642. [胡庆芳, 曹士地, 杨辉斌等. 汉江流域安康站日径流预测的 LSTM 模型初步 研究. 地理科学进展, 2020, 39(4): 636-642.]

[27] Zhou YL, Guo SL, Zhang FZ et al. Hydrological forecasting using artificial intelligence techniques. Journal of Water Resources Research, 2019, 8(1) : 1-12. DOI: 10.12677/JWRR.2019.81001. [周研来, 郭生练, 张斐章等. 人工智能在 水文预报中的应用研究. 水资源研究, 2019, 8(1): 1-12.]

[28] Fan HX, Jiang ML, Xu LG et al. Comparison of long short term memory networks and the hydrological model in runoff simulation. Water, 2020, 12(1) : 175. DOI: 10.3390/w12010175.

[29] Fan HX, He HD, Xu LG et al. Simulation and attribution analysis based on the long-short-term-memory network for detecting the dominant cause of runoff variation in the Lake Poyang Basin. J Lake Sci, 2021, 33 (3) : 866-878. DOI: 10. 18307/2021.0319. [范宏翔, 何菡丹, 徐力刚等. 基于长短记忆模型的鄱阳湖流域径流模拟及其演变的归因分析. 湖泊科学, $2021,33(3)$ : 866-878. DOI: 10.18307/2021.0319.]

[30] Gong R, He Y, Xu LG et al. The application progress of environmental fluid dynamics code( EFDC) in lake and reservoir environment. Transactions of Oceanology and Limnology, 2016, (6): 12-19. [龚然, 何跃, 徐力刚等. EFDC( Environmental Fluid Dynamics Code) 模型在湖库水环境模拟中的应用进展. 海洋湖沼通报, 2016, (6) : 12-19.]

[31] Hamrick JM. A Three-Dimensional Environmental Fluid Dynamics Computer Code: Theoretical and computational aspects. The College of William and Mary, Virginia Institute of Marine Science, Special Report 317, 1992: 63.

[32] Blumberg AF, Mellor GL. A description of a three-dimensional coastal ocean circulation model. Three-Dimensional Coastal Ocean Models. Washington, D. C.: American Geophysical Union, 1987: 1-16. DOI: 10.1029/co004p0001. 
[33] Johnson BH, Kim KW, Heath RE et al. Validation of three-dimensional hydrodynamic model of Chesapeake bay. Journal of Hydraulic Engineering, 1993, 119(1) : 2-20. DOI: 10.1061/(asce)0733-9429(1993)119: 1(2).

[34] Yao J, Zhang Q, Li YL et al. The influence of uniform winds on hydrodynamics of Lake Poyang. J Lake Sci, 2016, 28 (1) : 225-236. DOI: 10.18307/2016.0126. [姚静, 张奇, 李云良等. 定常风对鄱阳湖水动力的影响. 湖泊科学, $2016,28(1): 225-236$. ]

[35] Guo H, Su BD, Wang YJ et al. Runoff coefficients change and the analysis of the relationship between climate factors and runoff coefficients in Poyang Lake Basin (China) : 1955-2002. J Lake Sci, 2007, 19(2) : 163-169. DOI: 10.18307/ 2007.0209. [ 郭华, 苏布达, 王艳君等. 鄱阳湖流域 1955-2002 年径流系数变化趋势及其与气候因子的关系. 湖 泊科学, 2007, 19(2): 163-169.]

[36] Fan HX, Xu LG, Tao $\mathrm{H}$ et al. Accessing the difference in the climate elasticity of runoff across the Poyang Lake basin, China. Water, 2017, 9(2) : 135. DOI: 10.3390/w9020135.

[37] Wang XL, Han JY, Xu LG et al. Soil characteristics in relation to vegetation communities in the wetlands of Poyang Lake, China. Wetlands, 2014, 34(4) : 829-839. DOI: 10.1007/s13157-014-0546-x.

[38] Gassman PW, Sadeghi AM, Srinivasan R. Applications of the SWAT model special section: Overview and insights. Journal of Environmental Quality, 2014, 43(1) : 1-8. DOI: 10.2134/jeq2013.11.0466.

[39] Cao WZ, Hong HS, Yue SP. Modelling agricultural nitrogen contributions to the Jiulong River estuary and coastal water. Global and Planetary Change, 2005, 47(2/3/4) : 111-121. DOI: 10.1016/j.gloplacha.2004.10.006.

[40] Li YL, Zhang Q, Werner AD et al. Investigating a complex lake-catchment-river system using artificial neural networks: Poyang Lake (China). Hydrology Research, 2015, 46(6) : 912-928. DOI: 10.2166/nh.2015.150.

[41] Shen CP. A transdisciplinary review of deep learning research and its relevance for water resources scientists. Water Resources Research, 2018, 54(11) : 8558-8593. DOI: 10.1029/2018WR022643.

[42] Shen CP, Laloy E, Elshorbagy A et al. HESS Opinions: Incubating deep-learning-powered hydrologic science advances as a community. Hydrology and Earth System Sciences, 2018, 22(11) : 5639-5656. DOI: 10.5194/hess-22-5639-2018.

[43] Shen ZY, Chen XF, Zhao X et al. The influence of parameter uncertainty on phosphorus modeling: A case study of EFDC model application to the Three Gorges Reservoir Region, China. Fresenius Environmental Bulletin, 2014, 23 ( 1 ) : 264-273.

[44] Kratzert F, Klotz D, Brenner C et al. Rainfall-runoff modelling using Long Short-Term Memory ( LSTM) networks. Hydrology and Earth System Sciences, 2018, 22(11) : 6005-6022. DOI: 10.5194/hess-22-6005-2018.

[45] Qian GW, You L, Chen AM. Prediction of future water resources quantity in Poyang lake basin. Journal of Anhui Agricultural Sciences, 2017, 45(8) : 53-54, 73. [ 钱贵伍, 游黎, 陈爱民. 鄱阳湖流域水资源量预测. 安徽农业科学, 2017, $\mathbf{4 5}(8): 53-54,73$.

[46] Cheng JX, Xu LG, Jiang JH et al. Changes in streamflow at the outlet of dongting lake and their impacts on ecosystems. Resources and Environment in the Yangtze Basin, 2019, 28(5): 1225-1234. DOI: 10.11870/cjlyzyyhj201905022. [程俊 翔, 徐力刚, 姜加虎等. 洞庭湖出口径流变化及对生态系统的影响. 长江流域资源与环境, 2019, 28(5): 1225-1234.]

[47] Dai XZ, Luo BH. From “harnessing” to “enrichment” — research of strategic upgrading of mountain-river-lake engineering: Providing strong support for development of Changjiang Economic Zone. Yangtze River, 2016, 47(24) : 1-5, 11. [戴 星照, 罗斌华. 从“治”到“富”: “山江湖工程”战略升级研究. 人民长江, 2016, 47(24) : 1-5,11.]

[48] Yao W, Yu QH, You ZQ. Study on reservoirs operation at upper Yangtze River for alleviating low water situation of Poyang Lake. Yangtze River, 2015, 46(24): 1-5, 20. [要威, 余启辉, 游中琼. 长江上游水库调度缓解鄱阳湖枯水情势作 用研究. 人民长江, $2015,46(24): 1-5,20$.]

[49] Lu BH, Li KP, Zhang HW et al. Study on the optimal hydropower generation of Zhelin reservoir. Journal of Hydroenvironment Research, 2013, 7(4) : 270-278. DOI: 10.1016/j.jher.2013.01.002. 